\title{
Article \\ The Modulation of SCO2730/31 Copper Chaperone/Transporter Orthologue Expression Enhances Secondary Metabolism in Streptomycetes
}

\author{
Nathaly González-Quiñónez ${ }^{1}$, Ignacio Gutiérrez-Del-Río ${ }^{1} @$, Paula García-Cancela ${ }^{2}$, Gemma Fernández-García ${ }^{1}$, \\ Sergio Alonso-Fernández ${ }^{1}\left(\mathbb{D}\right.$, Paula Yagüe ${ }^{1}$, Álvaro Pérez-Valero ${ }^{1}\left[\right.$, María Montes-Bayón ${ }^{2}$, Felipe Lombó ${ }^{1}(\mathbb{D}$ \\ and Ángel Manteca 1,*iD \\ 1 Área de Microbiología, Departamento de Biología Funcional, IUOPA, ISPA, Facultad de Medicina, \\ Universidad de Oviedo, 33006 Oviedo, Spain; natygq@gmail.com (N.G.-Q.); \\ nachogutiem@gmail.com (I.G.-D.-R.); gemmafg06@hotmail.com (G.F.-G.); \\ sergioalonsofernandez@gmail.com (S.A.-F.); yaguepaula@uniovi.es (P.Y.); apv.moratalla@gmail.com (Á.P.-V.); \\ lombofelipe@uniovi.es (F.L.) \\ 2 Department of Physical and Analytical Chemistry, ISPA, Faculty of Chemistry, Universidad de Oviedo, \\ 33006 Oviedo, Spain; garciacpaula@uniovi.es (P.G.-C.); montesmaria@uniovi.es (M.M.-B.) \\ * Correspondence: mantecaangel@uniovi.es
}

check for updates

Citation: González-Quiñónez, N.; Gutiérrez-Del-Río, I.; García-Cancela, P.; Fernández-García, G.; Alonso-Fernández, S.; Yagüe, P.; Pérez-Valero, Á.; Montes-Bayón, M.; Lombó, F.; Manteca, Á. The Modulation of SCO2730/31 Copper Chaperone/Transporter Orthologue Expression Enhances Secondary Metabolism in Streptomycetes. Int. J. Mol. Sci. 2021, 22, 10143. https:// doi.org/10.3390/ijms221810143

Academic Editor: Jan Kormanec

Received: 15 August 2021

Accepted: 17 September 2021

Published: 20 September 2021

Publisher's Note: MDPI stays neutral with regard to jurisdictional claims in published maps and institutional affiliations.

Copyright: (c) 2021 by the authors. Licensee MDPI, Basel, Switzerland. This article is an open access article distributed under the terms and conditions of the Creative Commons Attribution (CC BY) license (https:/ / creativecommons.org/licenses/by/ $4.0 /)$.

\begin{abstract}
Streptomycetes are important biotechnological bacteria that produce several clinically bioactive compounds. They have a complex development, including hyphae differentiation and sporulation. Cytosolic copper is a well-known modulator of differentiation and secondary metabolism. The interruption of the Streptomyces coelicolor SCO2730 (copper chaperone, SCO2730::Tn5062 mutant) blocks SCO2730 and reduces SCO2731 (P-type ATPase copper export) expressions, decreasing copper export and increasing cytosolic copper. This mutation triggers the expression of 13 secondary metabolite clusters, including cryptic pathways, during the whole developmental cycle, skipping the vegetative, non-productive stage. As a proof of concept, here, we tested whether the knockdown of the SCO2730/31 orthologue expression can enhance secondary metabolism in streptomycetes. We created a SCO2730/31 consensus antisense mRNA from the sequences of seven key streptomycetes, which helped to increase the cytosolic copper in S. coelicolor, albeit to a lower level than in the SCO2730::Tn5062 mutant. This antisense mRNA affected the production of at least six secondary metabolites (CDA, 2-methylisoborneol, undecylprodigiosin, tetrahydroxynaphtalene, $\alpha$-actinorhodin, $\varepsilon$-actinorhodin) in the S. coelicolor, and five (phenanthroviridin, alkylresorcinol, chloramphenicol, pikromycin, jadomycin $\mathrm{G}$ ) in the $S$. venezuelae; it also helped to alter the $S$. albus metabolome. The SCO2730/31 consensus antisense mRNA designed here constitutes a tool for the knockdown of SCO2730/31 expression and for the enhancement of Streptomyces' secondary metabolism.
\end{abstract}

Keywords: Streptomyces; differentiation; copper; secondary metabolism

\section{Introduction}

Streptomycetes are important biotechnological bacteria, producing two thirds of the bioactive secondary metabolites currently used in clinical applications (mainly antibiotics, but also antitumourals, immunosuppressors, etc.) [1,2]. They have complex developmental cycles that include programmed cell death (PCD), hyphae differentiation and sporulation $[3,4]$.

Secondary metabolism in streptomycetes is highly regulated. There are regulators of specific secondary metabolism pathways as well as pleiotropic regulators affecting the production of several secondary metabolites [5]. There are also numerous predicted secondary metabolism pathways that have not been activated in the laboratory (cryptic pathways) [6,7]. Secondary metabolism is tightly connected with hypha differentiation and sporulation $[8,9]$, which makes Streptomyces' secondary metabolism even more complex. 
Understanding secondary metabolism regulation is one of the main challenges in industrial microbiology, and one of our best chances to activate the expression of cryptic pathways in the laboratory [10].

Copper is a well-known modulator of Streptomyces differentiation (aerial mycelium and sporulation) and secondary metabolism (antibiotic production) [11-15]. Cytosolic copper is highly regulated by copper chaperones and transporters, whose genetic expression is tightly modulated [16]. In a recent study, we quantified cytosolic copper in hypha and single spores, revealing that cytosolic copper correlates with spore germination, hypha differentiation and secondary metabolism activation [13,17]. The interruption of the S. coelicolor SCO2730 copper chaperone ORF (SCO2730::Tn5062 mutant) blocks SCO2730 and reduces the expression of SCO2731 (P-type ATPase copper export transporter), increasing cytosolic copper during germination and in the substrate mycelium hyphae (vegetative mycelium) [13]. There is a correlation between cytosolic copper, secondary metabolism activation and sporulation: low cytosolic copper concentrations are present in the vegetative hyphae; slightly higher cytosolic copper concentrations are present in secondary metabolite producer hyphae; and the highest cytosolic copper levels are present in sporulating hyphae and dormant spores [13]. Cytosolic copper in the S. coelicolor SCO2730::Tn5062 mutant is always above the necessary threshold for the activation of secondary metabolism, generating a unique phenotype consisting of the production of secondary metabolites (undecylprodigiosin for instance) during its whole developmental cycle, including germination and substrate mycelium; in other words, this mutant lacks an authentic vegetative non-producing stage [13]. The SCO2730::Tn5062 mutant shows a dramatic enhancement in the expression of genes from 13 secondary metabolite clusters $(43.3 \%$ of all predicted secondary metabolites produced by this strain), including six genetic clusters that were predicted to participate in secondary metabolite biosynthesis, yet were never observed under the usual laboratory cultivation conditions (cryptic pathways) [13].

The aim of this work was to reproduce the phenotype of the S. coelicolor SCO2730::Tn5062 copper chaperone/transport mutant (activation of secondary metabolism [13]) in other streptomycetes. The SCO2730/31 genes are highly conserved in Streptomyces (80.6 and $80.7 \%$ average nucleotide identity, respectively, among S. coelicolor, S. griseus, S. avermitillis, S. lividans, Saccharopolyspora erythraea (formerly Streptomyces erythraeus), S. tsukubaensis and S. venezuelae) [13]. However, the SCO2730/31's surrounding regions are not conserved enough to design a mutagenesis protocol capable of knocking out the SCO2730/31 orthologues in any streptomycete. The construction of an SCO2730::Tn5062 orthologue mutant for each streptomycete would be difficult to make scalable in high-throughput screening (HTS) campaigns. In this work, we opted for the creation of an antisense mRNA against a consensus sequence obtained from the SCO2730/31 orthologues from the seven streptomycetes indicated above. We tested this antisense mRNA in S. coelicolor, the Streptomyces model strain characterised by the production of coloured actinorhodin (blue) and undecylprodigiosin (red) antibiotics [18]; S. venezuelae, the strain from which chloramphenicol was discovered [19]; and S. albus, a strain widely used as a heterologous host [20]. The antisense SCO2730/31 consensus mRNA created here was successful in the modification of secondary metabolism in both Streptomyces species and constitutes a tool for knockdown SCO2730/31 orthologue expression in order to enhance secondary metabolism in streptomycetes.

\section{Results}

2.1. Construction of the S. coelicolor, S. venezuelae and S. albus SCO2730/31 Orthologue Knockdown Strains

We designed an antisense mRNA against a consensus sequence of the $S$. griseus, S. avermitillis, S. lividans, S. clavuligerus, Saccharopolyspora erythraea, S. tsukubaensis and S. venezuelae SCO2730/31 orthologues consisting of 2518 bp (Figure 1a-d, Figure S1). This sequence included the SCO2730 and SCO2731 ORF orthologues (Figure 1a,b) as well as two consensus sequences upstream of the SCO2730/31 orthologue ORFs, long enough to include the gene ribosomal binding sites (Figure 1c,d). The homology of the SCO2730/31 orthologue ORF sequences was over $50 \%$ for most of the consensus sequence positions 
(Figure 1a,b), which was considerably higher than the homology of the RBS sequences (Figure 1c,d). As a control, we also designed the S. coelicolor SCO2730/31 sequence that was $100 \%$ homologous to the $S$. coelicolor SCO2730/31 and their respective RBSs (2590 bps, Figure S1).

a
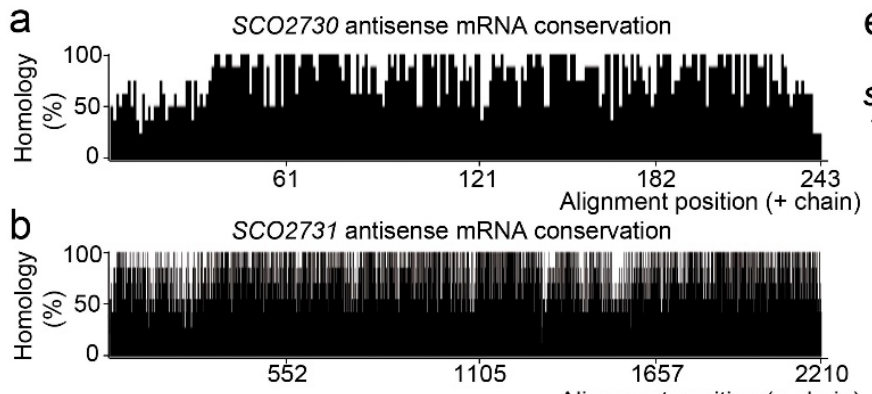

C SCO2730 RBS conservation

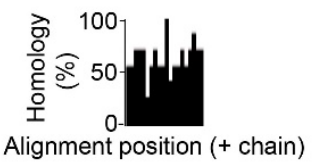

d SCO2731 RBS conservation

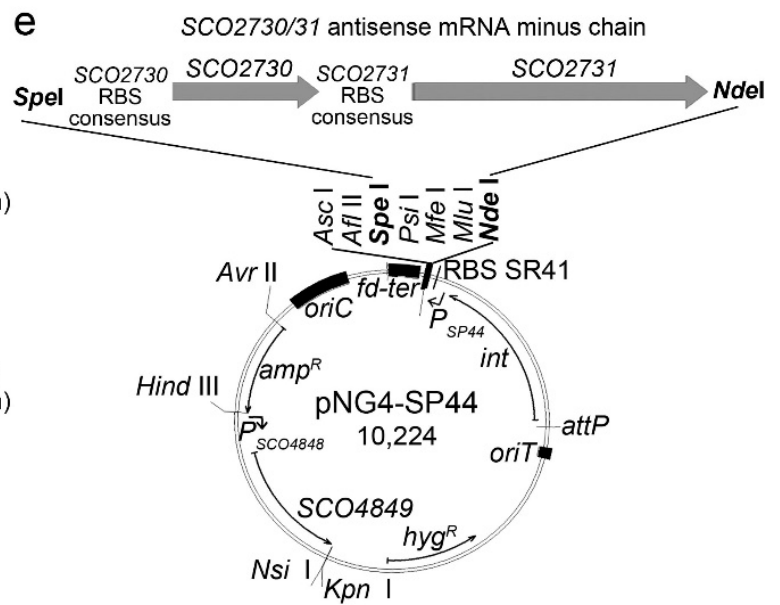

Figure 1. SCO2730/31 consensus antisense mRNA conservation and overexpression. (a-d) Histogram showing the percentages of homology of the SCO2730 and SCO2731 ORFs and the SCO2730 and SCO2731 RBSs. (e) Diagram showing the pNG4-SP44 overexpression plasmid controlling the expression of the SCO2730/31 antisense mRNA.

As detailed in the methods section, the SCO2730/31 antisense mRNA minus chain sequences were cloned into the $\Phi B T 1$ integrative pNG4-SP44 plasmid under the control of the SP44 strong constitutive promoter [21] (Figure 1e). Both constructions were used to knock down the SCO2730/31 gene expression in Streptomyces coelicolor. The pNG4-SP44 expressing the SCO2730/31 consensus antisense mRNA was also used to knock down the expression of the SCO2730/31 orthologues in S. venezuelae and S. albus.

\subsection{S. coelicolor SCO2730/31 Knockdown Mutants Affected Germination, Secondary Metabolite Production and Cytosolic Copper Levels}

The original SCO2730::Tn5062 copper chaperone/transport mutant, whose phenotype we wished to reproduce, showed as its main characteristics a delayed germination, a massive secondary metabolism activation and a high cytosolic copper accumulation [13]. To analyse the phenotypical effects of the antisense mRNAs, we compared the phenotype of the two S. coelicolor SCO2730/31 knockdown strains (those expressing the antisense mRNAs) against the SCO2730::Tn5062 mutant [13] and the S. coelicolor wild-type strain with and without the pNG4-SP44 plasmid (Figure 2).

Antibiotic production and germination were significantly affected in the SCO2730/31 knockdown mutants (Figure 2a,b). However, the effects in these phenotypes were different in the SCO273031 knockdown mutants and the original SCO2730::Tn5062 mutant. The knockdown mutants demonstrated a lower increase in antibiotic production (compared to the wild-type strain with and without the pNG4-SP44 plasmid) than the SCO2730::Tn5062 mutant (Figure 2a). The effect on antibiotic production was lesser in the SCO2730/31 knockdown mutant expressing the $S$. coelicolor antisense mRNA (100\% homologue to $S$. coelicolor) than in the SCO2730/31 knockdown mutant expressing the consensus mRNA; there was no significant increase in actinorhodin production in the latter mutant (Figure 2a). Germination was reduced in both SCO2730/31 knockdown mutants compared to the wildtype strain with and without pNG4-SP44, but the reduction was lower than that in the SCO2730::Tn5062 mutant (Figure 2b). 
a

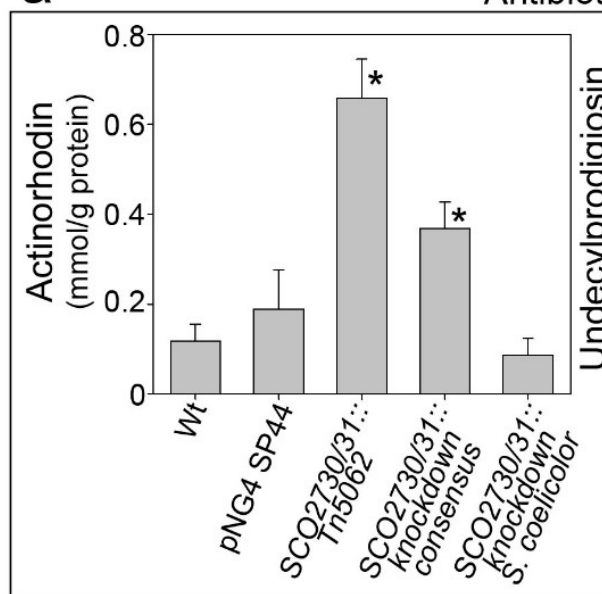

Antibiotic

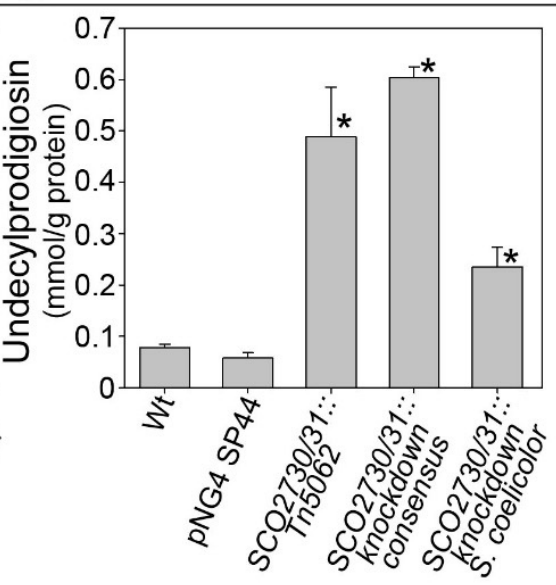

b

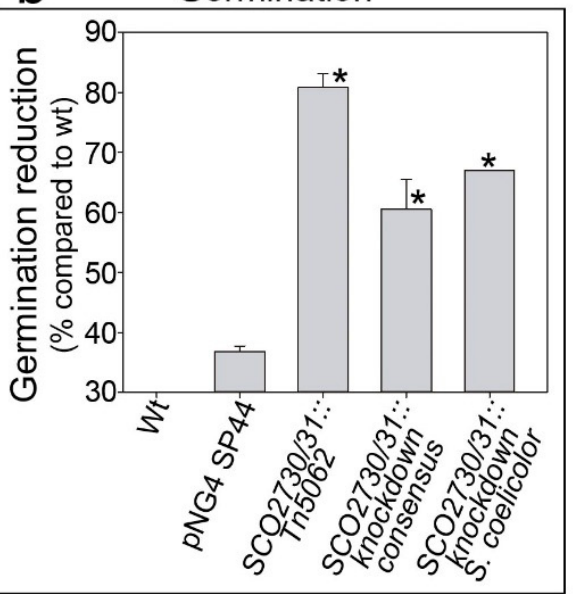

C

Cytosolic copper
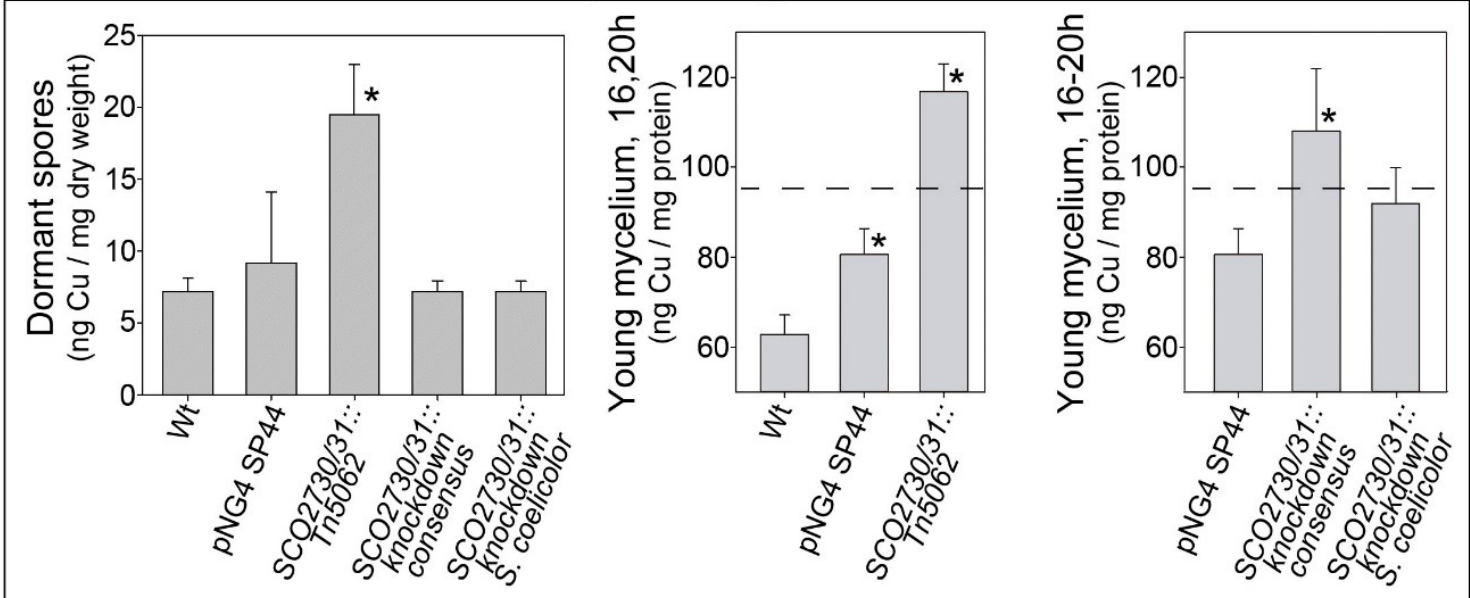

Figure 2. Antibiotic production, germination and cytosolic copper concentration in the S. coelicolor SCO2730/31 knockdown mutants compared to the wild-type strain with and without the pNG4-SP44 plasmid and the SCO2730::Tn5062 mutant. (a) Maximum actinorhodin and undecylprodigiosin production (145 h sucrose-free R5A liquid cultures). (b) Percentage of germination reduction compared to the wild-type strain (8 h GYM solid cultures). (c) Cytosolic copper in dormant spores (left panel, SFM cultures) and young mycelium (16-20 h sucrose-free R5A liquid cultures) (middle and right panels). The dashed line indicates the cytosolic copper umbral triggering secondary metabolism [13]. The asterisks indicate significant differences compared to the wild-type strain or the wild-type strain harbouring the pNG4-SP44 plasmid ( $p$ value equal to or less than 0.05).

As reported previously [13], cytosolic copper in dormant spores was significantly higher in the SCO2730::Tn5062 mutant compared to the wild-type strain (Figure 2c). However, there were no significant differences between the cytosolic copper in the spores of the knockdown mutants and in the wild-type strain (Figure 2c). Cytosolic copper in young hyphae was also higher in the SCO2730::Tn5062 mutant compared to the wild-type strain. Surprisingly, cytosolic copper in young hyphae was slightly increased in the wild-type strain harbouring the empty pNG4-SP44 plasmid (Figure 2c, middle panel), albeit to a considerably lower extent than in the SCO2730/31 knockdown mutant expressing the consensus mRNA (Figure 2c, right panel). The increase in cytosolic copper in the young hyphae of the SCO2730/31 knockdown mutant expressing the S. coelicolor antisense mRNA (100\% homologue to S. coelicolor) was not significant compared to the wild-type strain harbouring the pNG4-SP44 plasmid (Figure 2c, right panel). 
2.3. SCO2730/31 Expression Knockdown had a Large Effect on the S. coelicolor Metabolome, Albeit to a Lesser Extent than the Effect Observed in the SCO2730::Tn5062 Mutant

As the increase in actinorhodin and undecylprodigiosin production of the SCO2730/31 antisense consensus mRNA were higher than the effect of the S. coelicolor SCO2730/31 antisense mRNA (Figure 2a), the detailed metabolome analyses were performed only in the $S$. coelicolor strain overexpressing the SCO2730/31 antisense consensus mRNA. We compared the metabolomes of the S. coelicolor wild-type strain against the $S$. coelicolor harbouring the empty pNG4-SP44 plasmid (pNG4-SP44 strain), the S. coelicolor harbouring pNG4-SP44 expressing the consensus SCO2730/31 antisense RNA (SCO2730/31 knockdown strain) and the original SCO2730::Tn5062 mutant [13]. The results of the comparison of the metabolomes of three biological replicates, obtained at $145 \mathrm{~h}$ from sucrose-free liquid R5A cultures (the time-point at which maximum antibiotic production was reached), are summarised in Figure 3. In total, 432 compounds showed significant differences in the S. coelicolor strain harbouring the empty pNG44-SP44 plasmid compared to the wild-type strain (q-value less than 0.05 and fold change over 2.5-fold); 1699 compounds showed significant differences between the wild-type strain and the S. coelicolor SCO2730/31 knockdown; and 7448 compounds showed significant differences between the wild-type strain and the SCO2730::Tn5062 mutant (Figure 3a, Table S1).
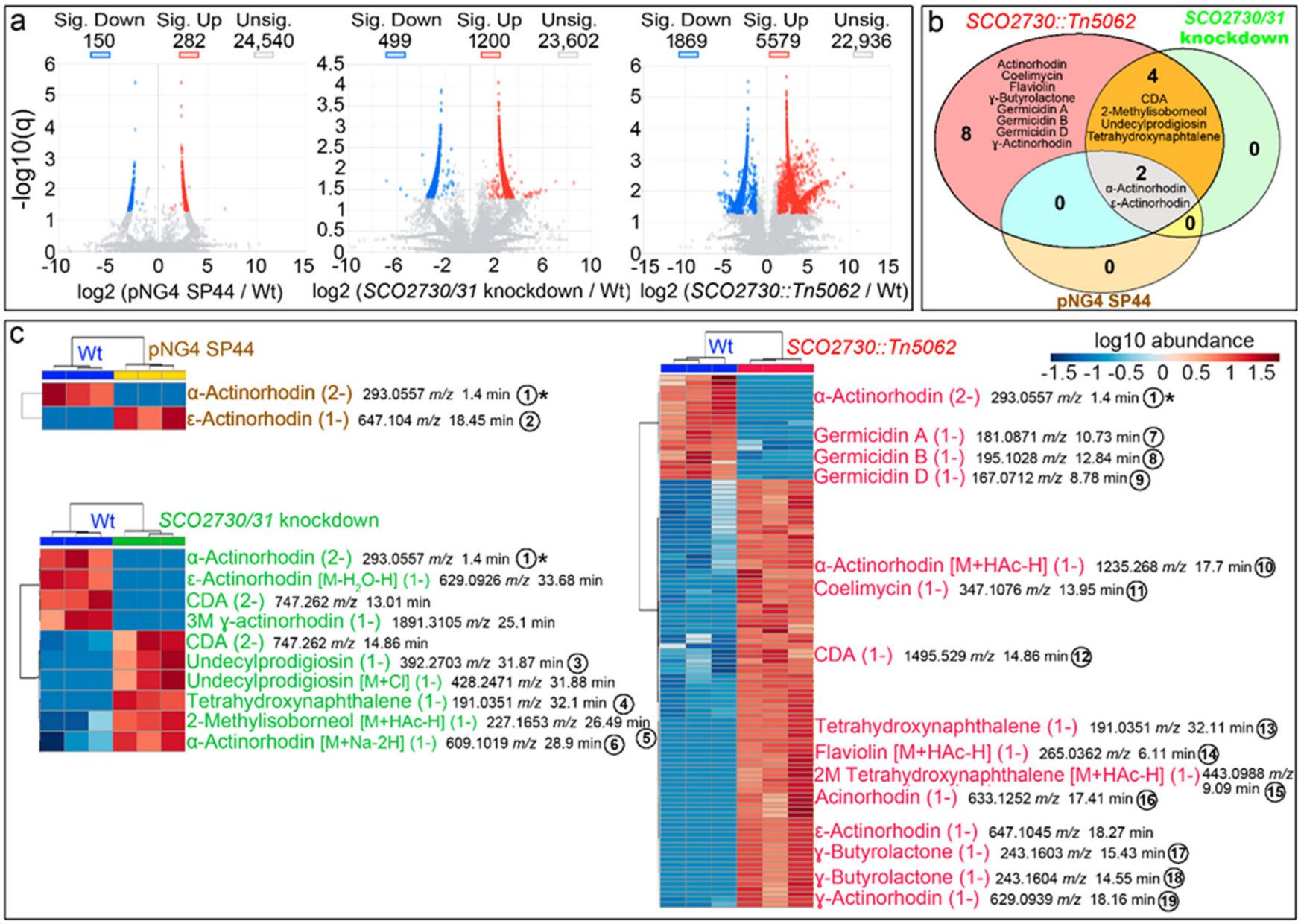

Figure 3. Metabolome analysis of the S. coelicolor SCO2730::Tn5062 and the SCO2730/31 knockdown mutants compared to the S. coelicolor wild-type strain with and without pNG4-SP44. (a) Volcano plots of the abundance values of the compounds detected in each strain compared to the wild-type strain. Significant up- (red) and down-regulated (blue) compounds are 
indicated. (b) Venn diagram showing the putatively identified secondary metabolites, showing significantly affected production in the SCO2730::Tn5062 mutant, the SCO2730/31 knockdown mutant and the pNG4-SP44 strains compared to the wild-type strain. (c) Heat-map showing the abundance values of putatively identified secondary metabolite adducts and isomers showing significant differences compared to the wild-type strain. The abundance values from three biological replicates are shown. The numbers indicate compounds detected in only one of the samples, whose chromatograms are indicated in Figure 4. The asterisk indicates the only compound identified in all the strains that was significantly down-regulated compared to the wild-type strain.
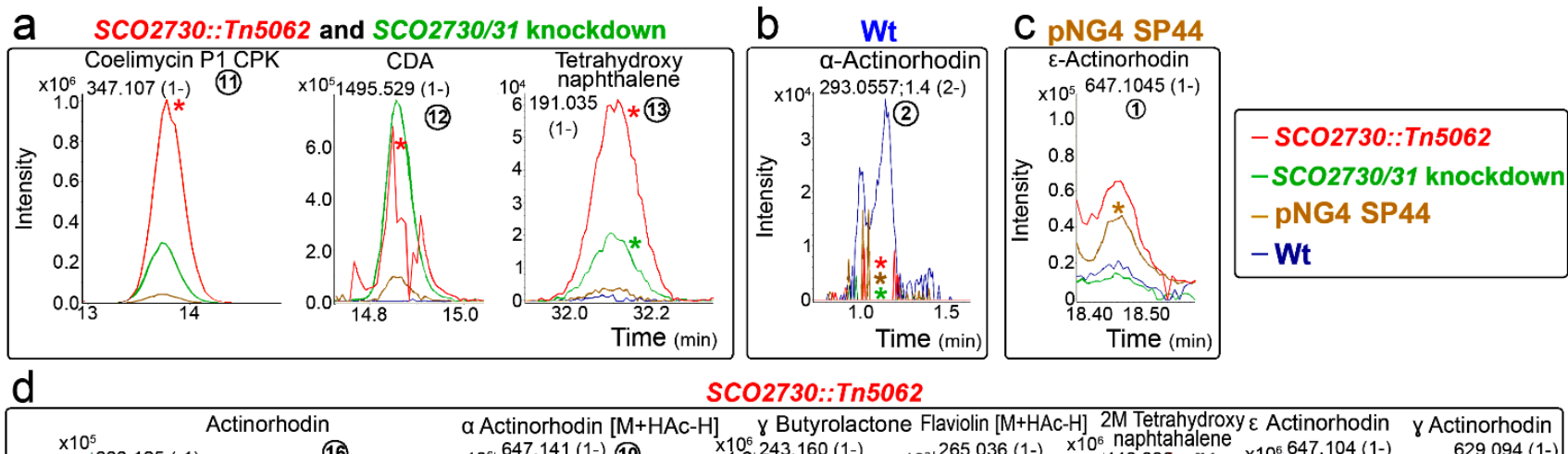

SC02730::Tn5062
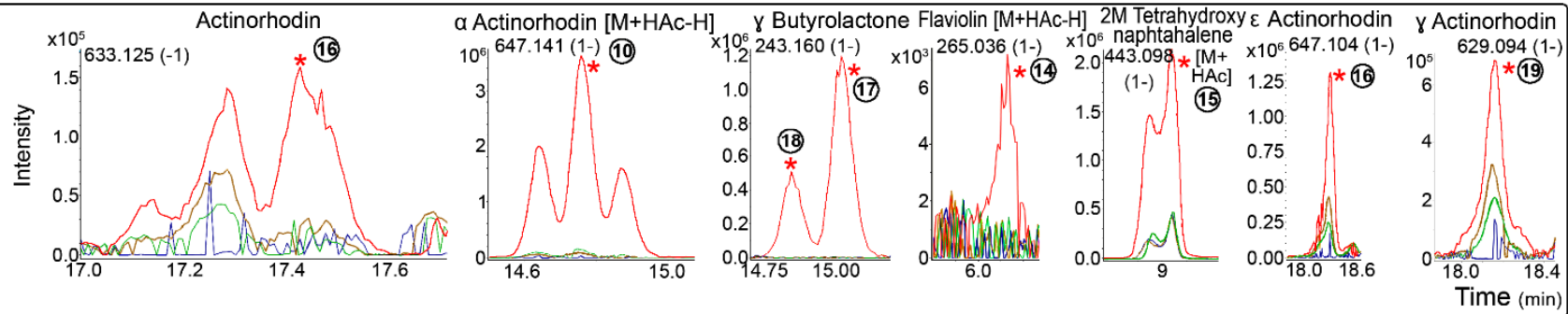

e

SCO2730/31 knockdown

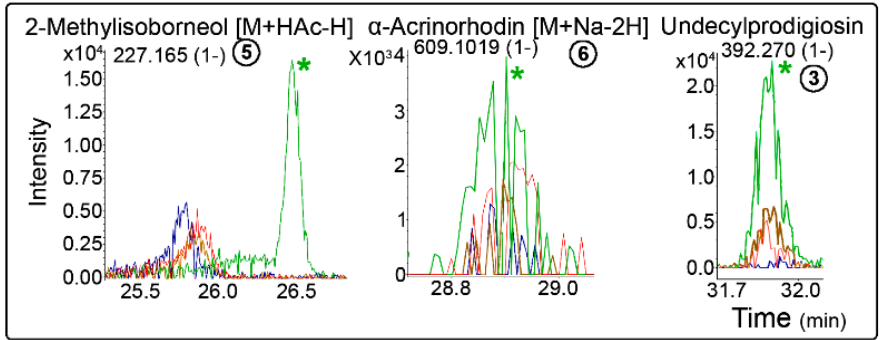

f Wt, pNG4 SP44 and SCO2730/31 knockdown

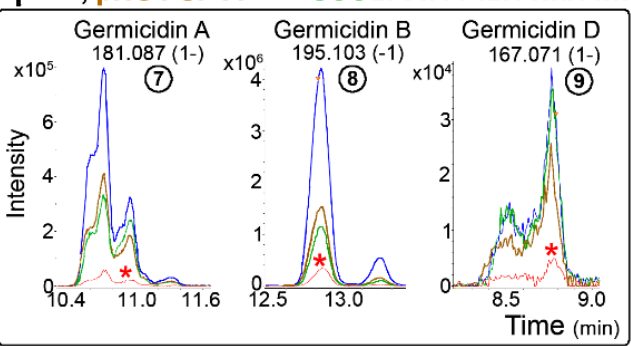

Figure 4. HPLC-MS chromatograms of some of the secondary metabolite compounds, showing different abundance in the S. coelicolor SCO2730::Tn5062 and the SCO2730/31 knockdown mutants compared to the S. coelicolor wild-type strain with and without pNG4-SP44. (a) Compounds showing higher abundances in the SCO2730::Tn5062 and the SCO2730/31 knockdown mutants. (b) Compound showing higher abundance in the S. coelicolor wild-type strain. (c) Compound showing higher abundance in the S. coelicolor wild-type strain harbouring pNG4-SP44. (d) Compounds showing higher abundances in the SCO2730::Tn5062 mutant. (e) Compounds showing higher abundances in the SCO2730/31 knockdown mutant. (f) Compounds showing higher abundances in the S. coelicolor wild-type strain with and without pNG4-SP44, and the SCO2730/31 knockdown mutant. Only one biological replicate is shown. The asterisks indicate significant differences (compared to the wild-type strain). The numbers are as in Figure 3.

Among the compounds showing significant differences between the S. coelicolor SCO2730/31 mutants analysed and the wild-type strain, we identified, based on the bibliography [22] and their exact masses ( \pm 0.001 Da maximum allowed variation), putative LC-MS $\mathrm{m} / \mathrm{z}$ ions from at least 14 secondary metabolites (Figure $3 \mathrm{~b}$ ): actinorhodin, coelimycin, flaviolin, $\gamma$-butyrolactone, germicidin A, germicidin B, germicidin $\mathrm{D}$, calcium-dependent antibiotic (CDA), 2-methylisoborneol, undecylprodigiosin, tetrahydroxynaphtalene, $\alpha$ actinorhodin, $\gamma$-actinorhodin and $\varepsilon$-actinorhodin (Figure $3 b$ ). The production of all these 14 compounds was affected in the SCO2730::Tn5062 mutant compared to the wild-type strain. CDA, 2-methylisoborneol, undecylprodigiosin and tetrahydroxynaphtalene produc- 
tion was altered in the $S$. coelicolor SCO2730/31 knockdown mutant; $\alpha$-and $\varepsilon$-actinorhodins were affected in the SCO2730::Tn5062 and SCO2730/31 knockdown mutants, as well as in the wild-type strain harbouring the pNG4-SP44 empty plasmid. Among the other eight compounds, secondary metabolite production was only affected in the SCO2730::Tn5062 mutant (Figure 3b).

A detailed analysis of the abundance of the specific isomers and adducts of the secondary metabolites putatively identified, showing significant differences between the S. coelicolor SCO2730/31 mutants and the wild-type strain, is shown in Figure 3c. The abundance of two compounds was affected in S. coelicolor [pNG4-SP44], 10 in the S. coelicolor SCO2730/31 knockdown mutant and 88 in the SCO2730::Tn5062 mutant (Table S1). Interestingly, the differences in secondary metabolism between the two SCO2730/31 mutants analysed and the wild-type strain were greater than those that can be inferred from Figure $3 b$. For instance, $\alpha$-actinorhodin showed significant differences in all strains compared to the wild-type strain (Figure $3 b$ ), but there was a specific adduct, $\alpha$ actinorhodin $(\mathrm{M}+\mathrm{Na}-2 \mathrm{H})(1-)$ (compound number 6 in Figure 3c), that was significantly overproduced in the SCO2730/31 knockdown strain and not in the SCO2730::Tn5062 mutant or the wild-type strain harbouring pNG4-SP44 (Figure 3c).

The chromatograms of some representative secondary metabolites are shown in Figure 4. Most of the identified secondary metabolites were significantly up-regulated in the SCO2730::Tn5062 mutant compared to the other strains: coelimycin (compound 11), CDA (compound 12), two isomers of tetrahydronaphtalene (compounds 13 and 15), actinorhodin (compound 16), $\alpha$-actinorhodin (2M+HAc-H) (1-) (compound 10), $\gamma$-butyrolactone (compounds 17 and 18), flaviolin (M+HAc-H)- (compound 14), $\varepsilon$-actinorhodin (compound 16) and $\gamma$-actinorhodin (compound 19) (Figure $4 \mathrm{a}, \mathrm{d}$ ). The abundance of one of the tetrahydronaphtalene isomers detected (compound 13), was significantly up-regulated in the SCO2730::Tn5062 and the SCO2730/31 knockdown mutants (Figure 4a). Interestingly, the abundance of coelimycin (compound 11) and CDA (compound 12), that were significantly up-regulated in the SCO2730::Tn5062 mutant (Figure 4a) seemed to be up-regulated in the SCO2730/31 knockdown mutant as well, but without sufficient reproducibility to be statistically significant. By contrast, some compounds were significantly down-regulated in the SCO2730::Tn5062 mutant (germicidins A, B and D, and compounds 7-9; Figure 4f). Other compounds were overproduced in the S. coelicolor SCO2730/31 knockdown mutant, such as 2-methylisobormeol (M+HAc-H) (1-) (compound 5), $\alpha$-actinorhodin $(\mathrm{M}+\mathrm{Na}-2 \mathrm{H})$ (1-) (compound 6) and undecylprodigiosin (compound 3) (Figure 4e). This last result coincided with the abundance of the undecylprodigiosin production quantified spectrophotometrically, which was slightly more abundant in the S. coelicolor SCO2730/31 knockdown mutant (expressing the consensus antisense mRNA) than in the SCO2730::Tn5062 mutant (Figure 2a). The production of a unique compound, $\varepsilon$-actinorhodin (compound 1), was significantly up-regulated in the S. coelicolor strain harbouring pNG4-SP44 (Figure 4c). The only compound identified as significantly down-regulated in all the analysed strains compared to the wild-type strain was $\alpha$-actinorhodin (compound 2; Figure 4b).

\subsection{The SCO2730/31 Expression Knockdown had a Big Effect on the S. venezuelae Metabolome}

Subsequently, we analysed the effect of the SCO2730/31 expression knockdown in $S$. venezuelae. We compared the metabolomes of the $S$. venezuelae wild-type strain against the $S$. venezuelae harbouring the empty pNG4-SP44 plasmid (pNG4-SP44 strain) and the S. venezuelae harbouring the pNG4-SP44 plasmid expressing the consensus SCO2730/31 antisense RNA (SCO2730/31 knockdown strain). The results of the metabolomes of three biological replicates of these strains, obtained at $48 \mathrm{~h}$ (the time point at which maximum antibiotic production was reached) from liquid MYM medium cultures [23] (supplemented with $2.1 \mathrm{~g} / \mathrm{L}$ MOPS), are shown in Figure 5. In total, 6438 compounds showed significant differences (q-value less than 0.05 and fold change greater than 2.5 -fold) in the S. venezuelae strain harbouring the empty pNG4-SP44 plasmid compared to the wild-type strain, whereas 4792 compounds showed significant differences between the S. venezuelae SCO3730/31 
knockdown and the wild-type strain. As discussed below, this result reveals a strong effect of the pNG4-SP44 plasmid on the S. venezuelae metabolome.
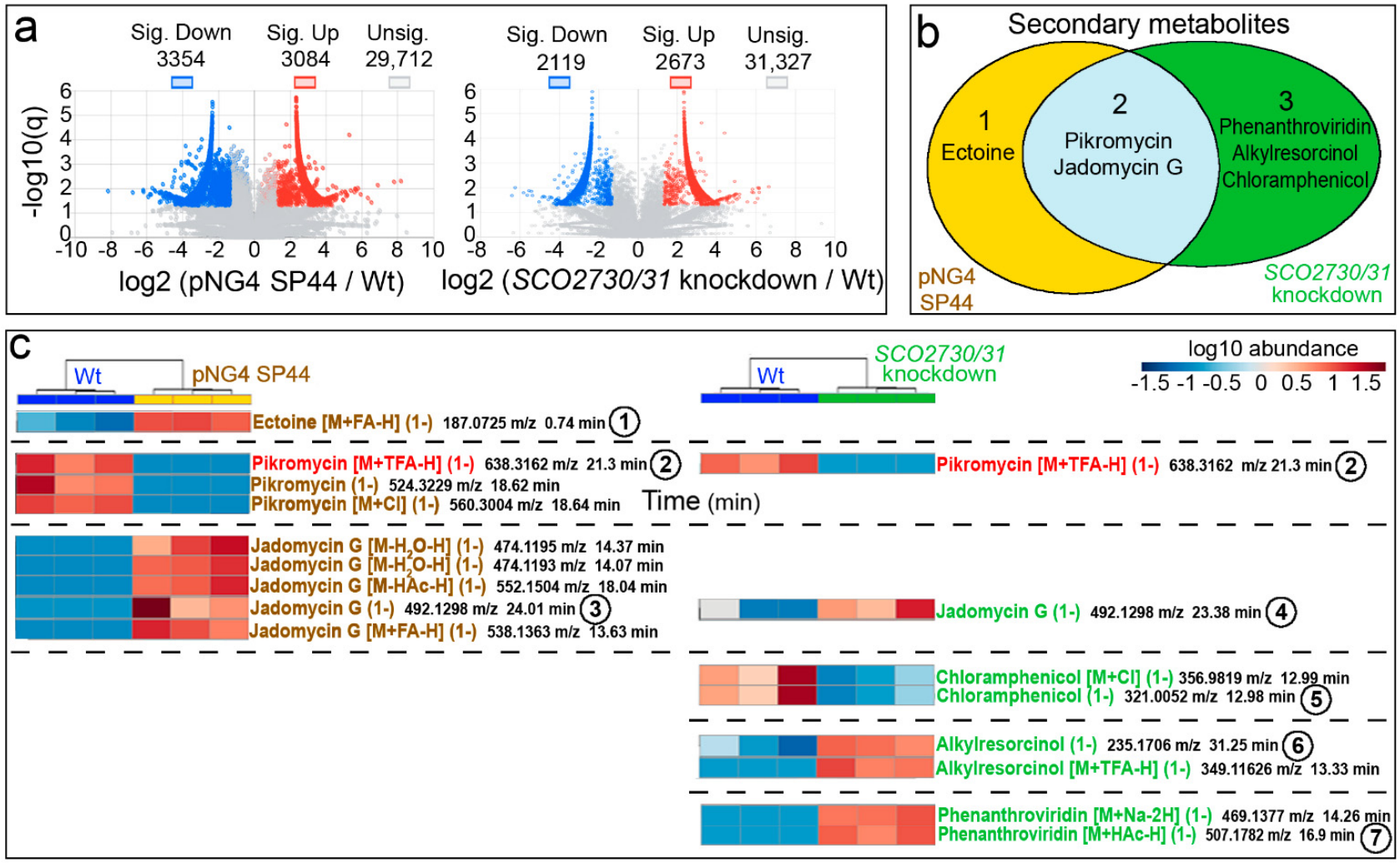

Figure 5. Metabolome analysis of the S. venezuelae SCO2730/31 knockdown mutant compared to the S. venezuelae wildtype-strain SP44. (a) Volcano plots of the abundance of the compounds detected in each strain compared to the wild-type strain. Significant up- (red) and down-regulated (blue) compounds are indicated. (b) Venn diagram showing the putatively identified secondary metabolites, showing significantly affected production in the SCO2730/31 knockdown mutant and the pNG4-SP44 strains compared to the wild-type strain. (c) Heat map showing the abundance values of the putatively identified secondary metabolite adducts and isomers, showing significant differences compared to the wild-type strain. The abundance values from three biological replicates are shown. The numbers indicate compounds whose chromatograms are indicated in Figure 6. The only adduct identified in the pNG4-SP44 and the SCO2730/31 strains is highlighted in red.

Among the compounds showing significant abundance differences in the S. venezuelae pNG4-SP44 and the S. venezuelae SCO2730/31 knockdown strains compared to the wild-type strain, we identified the LC-MS $m / z$ ions of six putative secondary metabolites: ectoine varied in the pNG4-SP44 strain; pikromycin and jadomycin G varied in both the pNG4SP44 and the SCO2730/31 knockdown strains; and phenanthroviridin, alkylresorcinol and chloramphenicol varied in the $S$. venezuelae SCO2730/31 knockdown strain (Figure $5 b$ ).

A detailed analysis of the abundance of the specific HPLC-MS secondary metabolite $m / z$ ions differentially produced in the pNG4-SP44 and the SCO2730/31 knockdown strains compared to the wild-type strain (Figure 5c; Table S2) revealed that the differences were higher than those that can be inferred from Figure $5 b$. Only one secondary metabolite was identified as differentially produced in both the pNG4-SP44 and the SCO2730/31 knockdown strains (pikromycin [M+TFA-H] (1-), compound 2, labelled in red in Figure 5c). The other 15 differential secondary metabolite ions were not shared between the pNG4SP44 (eight compounds) and the SCO2730/31 knockdown mutant (seven compounds) (Figure 5c). 

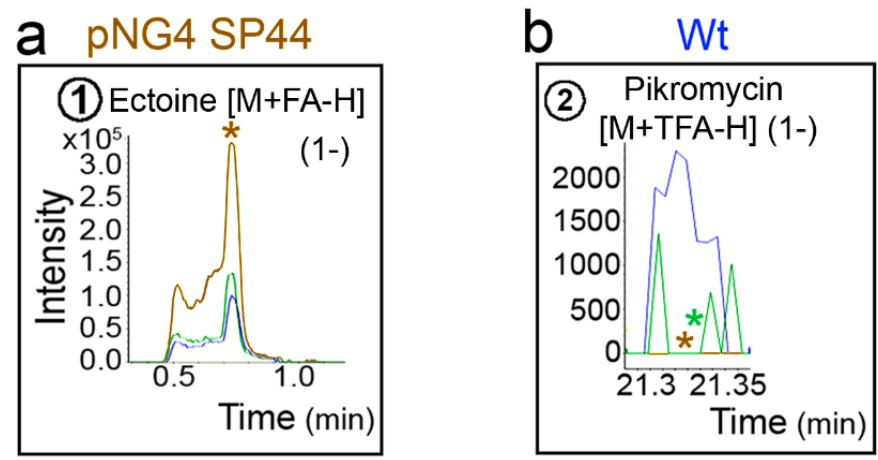

$$
\begin{aligned}
& \text { - SCO2730/31 } \\
& \text { knockdown } \\
& - \text { pNG4 SP44 } \\
& - \text { Wt }
\end{aligned}
$$
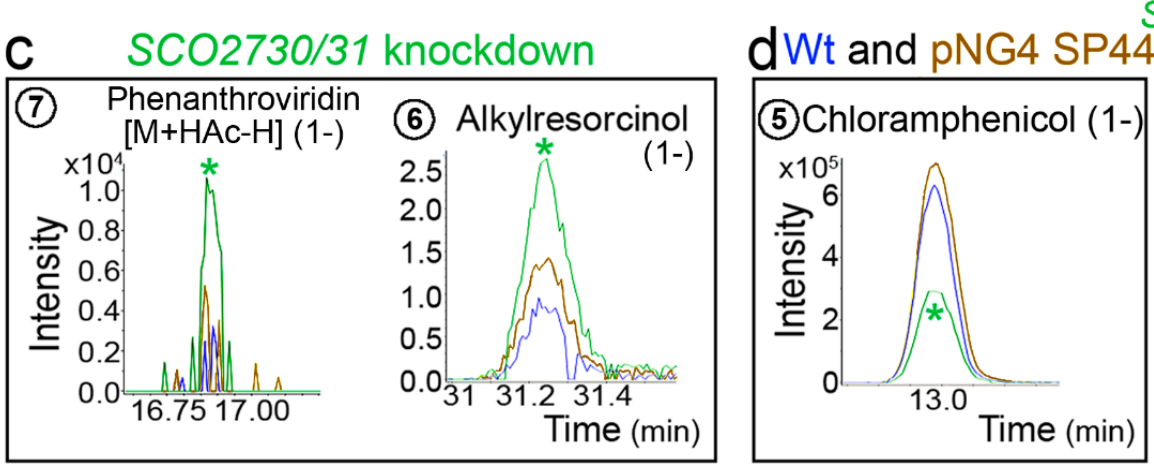

SC02730/31 knockdown

eor pNG4 SP44

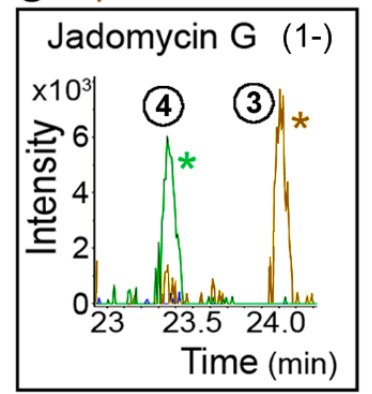

Figure 6. HPLC-MS chromatograms of some of the identified secondary metabolite compounds, showing different abundance values in the S. venezuelae SCO2730/31 knockdown mutant compared to the S. venezuelae wild-type strain with and without pNG4-SP44. (a) Compound showing higher abundance in the S. venezuelae strain harbouring pNG4-SP44. (b) Compound showing higher abundance in the $S$. venezuelae wild-type strain. (c) Compounds showing higher abundances in the S. venezuelae SCO2730/31 knockdown mutant. (d) Compounds showing higher abundances in the S. venezuelae wild-type strain with and without pNG4-SP44. (e) Jadomycin G isomers detected in the S. venezuelae strain harbouring pNG4-SP44 and the SCO2730/31 knockdown mutant. Only one biological replicate is shown. The asterisks indicate significant differences. The numbers are as in Figure 5.

Figure 6 shows the chromatograms of some of the HPLC-MS secondary metabolite $\mathrm{m} / \mathrm{z}$ ions differentially produced in the pNG4-SP44 and the SCO2730/31 knockdown strains compared to the wild-type strain. Ectoine [M+FA-H] (1-) was produced in higher amounts in the pNG4-SP44 strain (compound 1 in Figure 6a), whereas phenanthroviridin $[\mathrm{M}+\mathrm{HAc}-\mathrm{H}](1-)$ and alkylresorcinol were more abundant in the SCO2730/31 knockdown (compounds 6 and 7 in Figure 6c). Chloramphenicol production was also affected; in this case, it was reduced in the SCO2730/31 knockdown mutant but not in the strain harbouring the empty pNG4-SP44 plasmid (compound 5 in Figure 6d). The Jadomycin G chromatogram revealed that the same mass was identified at different retention times in the pNG4-SP44 (compounds 3 in Figure 6e) and the SCO2730/31 knockdown (compound 4 in Figure 6e) strains, indicating the presence of different isomers (Figure 6e). Pikromycin $(\mathrm{M}+\mathrm{TFA}-\mathrm{H})$ (1-) (compound 2) was the only compound whose production was reduced in both the $S$. venezuelae strains harbouring the pNG4-SP44 plasmid with and without the consensus antisense SCO2730/31 mRNA.

\subsection{The SCO2730/31 Expression Knockdown Modified the S. albus Metabolome}

Finally, we analysed the effect of the SCO2730/31 expression knockdown in S. albus. We compared the metabolomes of the $S$. albus wild-type strain against the $S$. albus harbouring the empty pNG4-SP44 plasmid (pNG4-SP44 strain) and the S. albus harbouring the pNG4-SP44 plasmid expressing the consensus SCO2730/31 antisense RNA (SCO2730/31 knockdown strain). In order to perform a quick analysis, the metabolomes were compared using high-performance liquid chromatography coupled with diode-array detection (HPLC-DAD) (Figure 7). The HPLC-DAD chromatograms were almost identical in the $S$. albus wild-type strain and the S. albus harbouring the empty pNG4-SP44 plasmid (Figure 7). 
However, there were several differences between the HPLC-DAD chromatograms of the wild-type and the S. albus SCO2730/31 knockdown strains (rectangles in Figure 7a, magnifications in Figure $7 \mathrm{~b}, \mathrm{c})$.
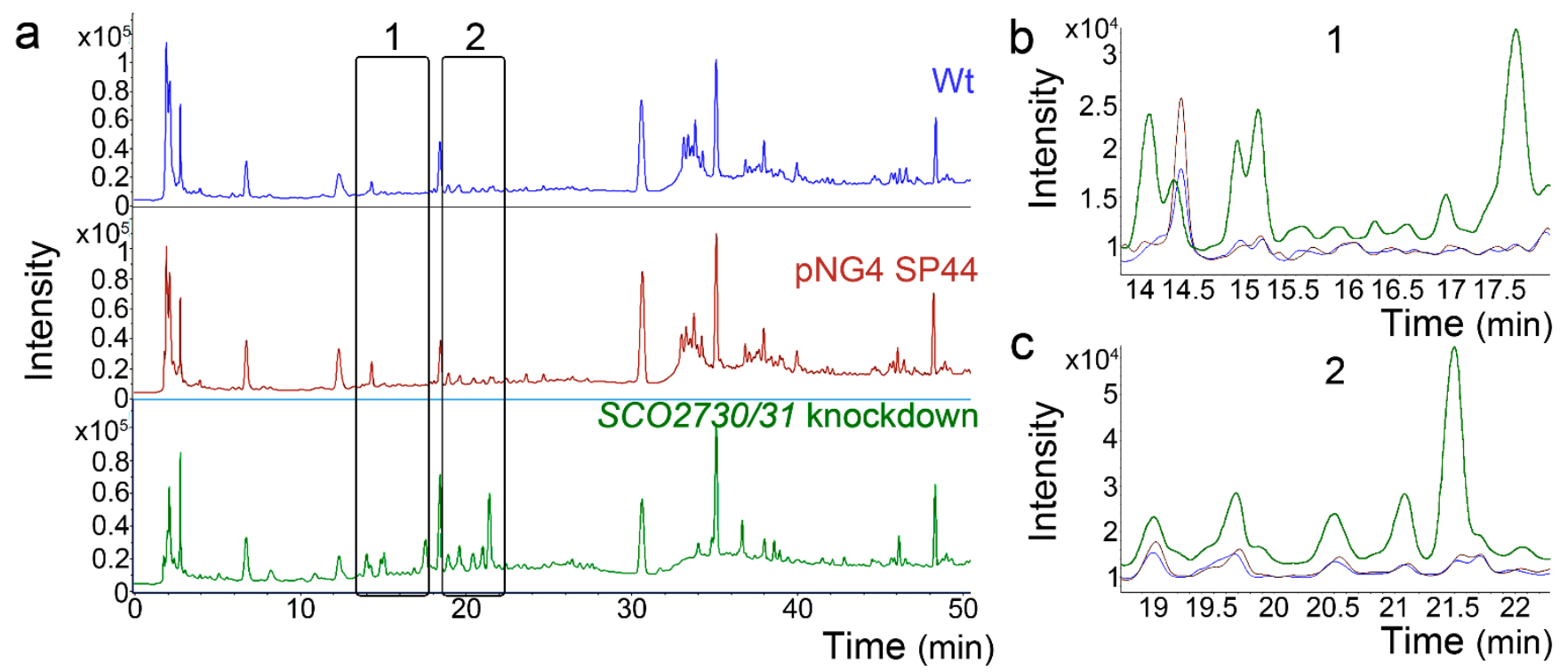

Figure 7. HPLC-DAD chromatograms of the S. albus SCO2730/31 knockdown mutant compared to the S. albus wild-type strain with and without pNG4-SP44. (a) Whole chromatograms. The rectangles mark the clearest differences between the SCO2730/31 knockdown mutant and the wild-type strain with or without pNG4-SP44. (b,c) Magnifications of the chromatogram areas marked with rectangles. Only one biological replicate is shown.

\section{Discussion}

As introduced above, the S. coelicolor SCO2730::Tn5062 mutant blocks the expression of the SCO2730 copper chaperone and reduces the expression of the SCO2731 copper transporter, augmenting cytosolic copper and increasing the transcription of 13 secondary metabolite clusters ( $43.3 \%$ of all predicted secondary metabolites produced by this strain) [13]. These secondary metabolites include six genetic clusters that were predicted to participate in secondary metabolite biosynthesis, yet were never observed under laboratory cultivation conditions (cryptic pathways) [13]. In this work, we performed a metabolomic analysis of this mutant, confirming that the activation of the transcription of secondary metabolite genes correlates with a massive increase in secondary metabolite production. The production of 88 putatively identified secondary metabolites was affected compared to the wild-type strain. Seventy-six of these compounds ( $86 \%$ of the total), were significantly more abundant in the mutant than in the wild-type strain (Figure 3c; Table S1).

The regulation of the $S C O 2730 / 31$ 's gene expression is highly complex and modulated by at least four promoters [13]. The SCO2730::Tn5062 mutant is interrupted at the beginning of the SCO2730 ORF, allowing a reduced expression of SCO2731 from a promoter located into SCO2102 [13]. Further unknown expression regulation networks cannot be discarded, since different combinations of the four promoters, together with the SCO2102/03 ORFs, cannot fully restore the SCO2730/31 transcription in the SCO2730::Tn5062 mutant [13]. The objective of this work was to reproduce the phenotype (secondary metabolism and cryptic pathway activation) of the SCO2730::Tn5062 mutant in other streptomycetes. To create the mutant in the corresponding SCO2730 orthologue for each streptomycete species would be tedious and unfeasible in high-throughput screening using several strains. Here, we designed a proof of concept consisting of a test of whether an antisense mRNA for a consensus sequence of the SCO2730/31 orthologue genes can successfully activate Streptomyces' secondary metabolism. The three S. coelicolor strains mutated in SCO2730/31 (SCO2730::Tn5062; SCO2730/31 knockdown expressing consensus antisense mRNA; SCO2730/31 knockdown expressing the $S$. coelicolor antisense mRNA) were different, and would conceivably pro- 
mote different SCO2730/31 copper chaperone/transporter doses. We could not perform a direct quantification of the SCO2730/31 protein amounts, but we quantified significant differences in cytosolic copper levels between the mutants and the wild-type strain (Figure 2c), which is an indirect indicator of the differences in the SCO2730/31 copper transporter activity. The differences in cytosolic copper lead to a different effect on actinorhodin and undecylprodigiosin production (Figure 2). These results support the copper dosedependent effect described by us in a previous study: secondary metabolism is activated in hypha harbouring cytosolic copper levels between 46 and $200 \mathrm{ng} \mathrm{Cu} / \mathrm{mg}$ protein, but it is inhibited at the high cytosolic copper levels reached during sporulation [13]. Theoretically, the antisense SCO2730/31 mRNA 100\% homologous to the S. coelicolor SCO2130/31 genes should hybridise more tightly to the SCO2730/31 mRNA than the consensus antisense mRNA, which is not identical to the S. coelicolor genes. However, the lower effect on cytosolic copper accumulation and antibiotic production of the former seems to indicate the contrary, i.e., a lower effect on the reduction of SCO2730/31 translation in S. coelicolor.

The S. coelicolor SCO2730/31 knockdown mutant overexpressing the consensus antisense mRNA significantly affected the production of 1699 compounds (Figure 3a), including 10 HPLC-MS $m / z$ ions from six putatively identified secondary metabolites (Figure $3 b, c)$. The antisense consensus mRNA also worked to modify the Streptomyces venezuelae metabolome (Figure 5). Although the empty pNG4-SP44 plasmid had a large effect on the $S$. venezuelae metabolome, significantly affecting the production of $6438 \mathrm{com}$ pounds (Figure $5 \mathrm{a}$ ), there were important differences between the $S$. venezulae harbouring pNG4-SP44 and the S. venezuelae knockdown mutant metabolomes, revealing that the consensus antisense mRNA also worked to alter secondary metabolism in S. venezuelae: of the six secondary metabolites from which an isomer or adduct was putatively identified, three (phenanthroviridin, alkylresorcinol and chloramphenicol) significantly affected production only in the strain expressing the consensus antisense mRNA (Figure 5b); pikromycin $(\mathrm{M}+\mathrm{TFA}-\mathrm{H})(1-)$ was the only adduct that was significantly altered in the S. venezuelae harbouring the pNG4-SP44 with and without the SCO2730/31 consensus antisense mRNA. The production of the other 16 HPLC-MS $m / z$ secondary metabolite ions putatively identified was different in the S. venezuelae SCO2730/31 knockdown mutant and the S. venezuelae strain harbouring the empty pNG4-SP44 plasmid.

The S. albus SCO2730/31 knockdown mutant overexpressing the consensus antisense mRNA showed an important alteration in its metabolome (Figure 7). Unlike the S. venezuelae, the empty pNG4-SP44 plasmid did not have a significant effect on the S. albus metabolome (Figure 7).

\section{Materials and Methods}

\subsection{Bacterial Strains and Culture Conditions}

All the Streptomyces and Escherichia coli strains used in this work are listed in Table S3.

The S. coelicolor spores were harvested from SFM solid plates [18] after growth at $30{ }^{\circ} \mathrm{C}$ for 7 days. The $S$. coelicolor germination was analysed in GYM [24] (5 g/L glucose, $4 \mathrm{~g} / \mathrm{L}$ yeast extract, $5 \mathrm{~g} / \mathrm{L}$ malt extract, $0.5 \mathrm{~g} / \mathrm{L} \mathrm{MgSO}_{4} \cdot 7 \mathrm{H}_{2} \mathrm{O}, 20 \mathrm{~g} / \mathrm{L}$ agar; after autoclaving supplemented with sterile $0.5 \mathrm{~g} / \mathrm{L} \mathrm{K}_{2} \mathrm{HPO}_{4}$ ) plates covered with cellophane, inoculated with $10^{7}$ spores from a freshwater suspension and cultured at $30 \mathrm{~m}{ }^{\circ} \mathrm{C}$. The fermentation was performed in liquid $50 \mathrm{~mL}$ sucrose-free R5A [25] cultures grown at $30^{\circ} \mathrm{C}$ and $200 \mathrm{rpm}$ in $250 \mathrm{~mL}$ flasks inoculated with $10^{7}$ spores $/ \mathrm{mL}$.

The $S$. venezuelae spores were harvested from MYM solid plates [23] after growth at $30^{\circ} \mathrm{C}$ for 8 days. The pre-inoculum for the fermentation was prepared using $5.10^{6}$ spores from a freshwater suspension in $10 \mathrm{~mL}$ of TSB medium (tryptic soy broth, Scharlau), and grown for $16 \mathrm{~h}$ at $30^{\circ} \mathrm{C}$ and $220 \mathrm{rpm}$ in $100 \mathrm{~mL}$ flasks. The fermentation was performed in $20 \mathrm{~mL}$ MYM medium supplemented with $2.1 \mathrm{~g} / \mathrm{L}$ MOPS and inoculated with $2 \mathrm{~mL}$ of pre-inoculum, at $30{ }^{\circ} \mathrm{C}$ and $220 \mathrm{rpm}$ in $250-\mathrm{mL}$ flasks. After $8 \mathrm{~h}$ of incubation, absolute ethanol was added to each culture to obtain a concentration of $6 \% v / v$. 
The S. albus spores were harvested from Bennett medium solid plates [18] after growth at $30{ }^{\circ} \mathrm{C}$ for 5 days. The fermentation was performed in liquid $50 \mathrm{~mL}$ NL333 medium [13] cultures grown at $30^{\circ} \mathrm{C}$ and $250 \mathrm{rpm}$ in $250 \mathrm{~mL}$ flasks inoculated with $10^{7}$ spores $/ \mathrm{mL}$.

The Escherichia coli strains were cultured in LB and 2xTY media at $37^{\circ} \mathrm{C}$. The following antibiotics were added to select plasmid-bearing and mutant strains: ampicillin $(100 \mu \mathrm{g} / \mathrm{mL})$, apramycin $(100 \mu \mathrm{g} / \mathrm{mL}$ for E. coli, $25 \mu \mathrm{g} / \mathrm{mL}$ for $S$. coelicolor $)$, hygromycin $(100 \mu \mathrm{g} / \mathrm{mL}$ for E. coli, $200 \mu \mathrm{g} / \mathrm{mL}$ for S. coelicolor $)$, kanamycin $(50 \mu \mathrm{g} / \mathrm{mL})$, chlorampheni$\operatorname{col}(25 \mu \mathrm{g} / \mathrm{mL})$ and nalidixic acid $(25 \mu \mathrm{g} / \mathrm{mL})$ (all from Invitrogen, Waltham, MA, USA).

\subsection{SCO2730/31 Bioinformatic Analyses and Antisense mRNA Design}

The SCO02730/2731 orthologue sequences and their surrounding genomic regions were analysed. The S. coelicolor, S. griseus, S. avermitillis, S. lividans and S. venezuelae orthologue sequences were obtained from the StrepDB (http://strepdb.streptomyces.org.uk/) (accessed on 10 June 2021). The Saccharopolyspora erythraea NRRL 2338 and S. tsukubaensis NRRL18488 SCO02730/2731 orthologue sequences were obtained from NCBI (genome accession numbers AM420293 and NZ_CP029159, respectively).

The SCO2730 orthologues were: SLI_3079 (S. lividans), SAV_5332 (S. avermitilis), SVEN_2533 (S. venezuelae), SGR_4828 (S. griseus), SACE_6610 (S. erythraea) and STSU_RS23720 (S. tsukubaensis).

The SCO2731 orthologues were: SLI_3080 (S. lividans), SAV_5331 (S. avermitilis), SVEN_2534 (S. venezuelae), SGR_4827 (S. griseus), SACE_6611 (S. erythraea) and STSU_RS20065 (S. tsukubaensis).

The sequences containing the putative SCO2730 and SCO2731 RBS sequences were obtained by collecting 20 nucleotides upstream of the ATG (or GTG) starting codon.

The SCO2730 and SCO2731 nucleotide similarities were estimated using the software package Lalign (http:/ / www.ch.embnet.org/software/LALIGN_form.html) (accessed on 10 June 2021).

The SCO2730, SCO2731 and RBSs' DNA sequences were aligned using the MUSCLE software available on the free online platform Phylemon (http:/ / phylemon.bioinfo.cipf.es/) (accessed on 10 June 2021). Ambiguous alignments in highly variable (gap-rich) regions were excluded from the antisense mRNA sequence (aligned sequences are available from the authors upon request).

The consensus antisense mRNA sequence $(2518 \mathrm{bps})$ was generated by fusing the consensus sequences of the four alignments (SCO2730, SCO2731 and the two RBS regions) (Figure S1). The homology plots shown in Figure 1a-c were created using the Jalview 2.11.0 software. A sequence 100\% homologous to the S. coelicolor SCO2730/31 and RBS sequences (2590 bps) was also designed (Figure S1). The synthesis of both sequences was ordered by BGI Genomics (Hong Kong).

\subsection{Overproduction of the SCO2730/31 Antisense mRNA}

The SCO2730/31 consensus and S. coelicolor antisense $(100 \%$ homologous to S. coelicolor) mRNAs were cloned, independently, in the pNG4 plasmid at the SpeI-NdeI cloning sites [13]. To increase the antisense mRNA production, the strong SP44 promoter [21] was cloned into this plasmid to control the overproduction of the antisense mRNA. In order to do this, we ordered the synthesis of the NdeI- fd-ter terminator-SP44-RBS SR41-NdeI DNA from BGI Genomics (Hong Kong). The fd-ter terminator [21] was included before the SP44 promoter to prevent its possible interference with the PermE * promoter present in the original pNG4 plasmid. The SR41 RBS [21] is not necessary for the expression of the antisense mRNA, but it was included in order to obtain the possibility of using this plasmid to overproduce proteins. The synthetised sequence was cloned into NdeI, and the correct orientation of the SP44 promoter, controlling the expression of the antisense mRNAs, was checked by XhoI digestion and confirmed by Sanger sequencing, using SP44F/R primers. The integration of the plasmid in the S. coelicolor and S. venezulae 
chromosomes was confirmed by PCR using the primers SCO4848F/pMS82R [26] and SCO4848F/vnz22340, respectively.

\subsection{Undecylprodigiosin and Actinorhodin Quantification}

Undecylprodigiosin and actinorhodin were quantified spectrophotometrically, in accordance with Tsao et al. [27] and Bystrykh et al. [28]. For actinorhodin quantification, $\mathrm{KOH}$ was added to the culture samples at a final concentration of $1 \mathrm{~N}$. The cellular pellets were discarded by centrifugation, and the actinorhodin concentration was spectrophotometrically determined at $640 \mathrm{~nm}$, applying the linear Beer-Lambert relationship $\left(\varepsilon_{640}=25,320\right)$. The culture samples for undecylprodigiosin quantification were lyophilised, resuspended in methanol, acidified with $0.5 \mathrm{~N} \mathrm{HCl}$ and spectrophotometrically assayed at $530 \mathrm{~nm}$, using the Beer-Lambert relationship to estimate concentrations $\left(\varepsilon_{530}=100,500\right)$. Three biological replicates were processed. The reliability of the differences in antibiotic production (compared to the wild-type strain) was analysed by Student's $t$-tests. Differences were considered significant if the $p$ value was equal to or less than 0.05 (asterisks in Figure 2a).

\subsection{Protein Quantification}

The protein concentration was quantified by Bradford [29], using bovine serum albumin standard (Sigma Aldrich, Burlington, MA, USA). The total protein extracts were obtained by mixing a volume of culture with a volume of $1 \mathrm{M} \mathrm{NaOH}$, boiling for $5 \mathrm{~min}$ and removing cell debris by centrifugation at $7740 \times g$.

\subsection{Cytosolic Copper Quantification in Dormant Spores and Mycelium}

Spores were obtained from SFM cultures. Young vegetative mycelium was obtained from sucrose-free R5A young cultures reaching a cellular mass between 0.009 and $0.012 \mathrm{mg}$ protein per $\mathrm{mL}$ ( 16 or $20 \mathrm{~h}$ culture depending on the specific strain). Cytosolic copper quantification was performed as reported previously [13]. Briefly, the spores or the mycelium were washed four times by centrifugation at $12,000 \times \mathrm{g}$ for $10 \mathrm{~min}$ at $4{ }^{\circ} \mathrm{C}$ and resuspended in a washing buffer (10 mM Tris- $\mathrm{HCl} \mathrm{pH} 7.5$ containing $1 \mathrm{mM}$ EDTA). The samples were centrifuged and washed in the washing buffer, but this time without EDTA. For the bulk analysis of $\mathrm{Cu}$ in the dormant spores, acid digestion was conducted by resuspending the spores in $65 \%$ sub-boiling purified $\mathrm{HNO}_{3}$ at $70{ }^{\circ} \mathrm{C}$ for $1 \mathrm{~h}$ and then $30 \% \mathrm{H}_{2} \mathrm{O}_{2}$ for $3 \mathrm{~h}$ at the same temperature. For the bulk analysis of $\mathrm{Cu}$ in the germinated spores and the mycelium, the samples were suspended in a rupture buffer $(10 \mathrm{mM}$ Tris- $\mathrm{HCl} \mathrm{pH} 7.5)$ The lysis step was performed using Fast-Prep (MP ${ }^{\mathrm{TM}}$ Biomedicals Germany $\mathrm{GmbH}$, Berlin, Germany) with six 20-s force 6.5 cycles and with $1 \mathrm{~min}$ on ice between each run. The cell debris was eliminated by centrifuging samples at $12,000 \times \mathrm{g}$ for $10 \mathrm{~min}$ at $4{ }^{\circ} \mathrm{C}$ and discarding the pellets. The resulting solutions were diluted with water and the total $\mathrm{Cu}$ content was determined by ICP-MS and referred to the dry mass of the spores $(1 \mathrm{~mL}$ of spores were washed with water, dried at $100{ }^{\circ} \mathrm{C}$ to a constant weight on pre-weighted tubes) or protein (measured with the Bradford assay).

All the measurements were conducted in the triple quadrupole-based ICP-MS Thermo iCAP-TQ (Thermo Fisher Scientific, Bremen, Germany) using the single quad mode and helium as collision gas. For the bulk analysis, the ICP was equipped with a Micro Mist nebuliser, a cyclonic spray chamber (both from ESI Elemental Service \& Instruments $\mathrm{GmbH}$, Mainz, Germany) and an auto-sampler ASX-560 (Teledyne CETAC Technologies, Omaha, NE, USA).

All the solutions were prepared using ultrapure water obtained from a Milli-Q system (Millipore, Bedford, MA, USA). The hydrogen peroxide for the acid digestion was obtained from Sigma-Aldrich (Saint Louis, MO, USA). The nitric acid (65\%, suprapur quality) was purchased from Merck Millipore (Darmstad, Germany) and further purified by sub-boiling distillation. The external calibrations were carried out with a Cu ICP standard CertiPur ${ }^{\circledR}$ $\left(1000 \mathrm{mgL}^{-1}\right)$, purchased from Merck. 
The reliability of the differences between the copper concentrations (compared to the wild-type strain) was analysed by Student's $t$-tests. The differences were considered significant if the $p$ value was equal to or less than 0.05 (asterisks in Figure 2c).

\subsection{Quantification of Spore Germination}

The S. coelicolor germination was quantified as previously described [13] in GYM [24] cellophane cultures inoculated with $10^{7}$ spores. Cellophane squares were manually cut, placed over a coverslip, stained with SYTO9 and propidium iodide (LIVE/DEAD BacLight Bacterial Viability Kit, Invitrogen, L1-3152) and observed under a Leica TCS-SP8 confocal laser-scanning microscope (Leica Microsistemas S.L.U., Spain) at wavelengths of 488 and $568 \mathrm{~nm}$ excitation and $530 \mathrm{~nm}$ (green) or $640 \mathrm{~nm}$ (red) emissions [30]. Three biological replicates were processed quantifying germination in at least 100 spores per replicate. Germination values were normalised as the percentage of germination reduction compared to the wild-type strain. The reliability of the differences in spore germination (compared to the wild-type strain) was analysed by Student's $t$-tests. The differences were considered significant if the $p$ value was equal to or less than 0.05 (asterisks in Figure 2).

\subsection{Compound Extraction and HPLC-MS Metabolome Analyses}

Three biological replicates were cultured and collected at the maximum secondary metabolite production time-points ( $145 \mathrm{~h}$ in the $S$. coelicolor strains; $48 \mathrm{~h}$ in the $S$. venezuelae strains). The cells and supernatants from the 20-mL cultures were separated by centrifugation at 10,000 rpm for $10 \mathrm{~min}$. In the case of the $S$. coelicolor, supernatant cultures and cells were processed, whereas in the $S$. venezuelae, only the supernatant cultures were processed. The supernatant culture compound extraction was carried out by adding 0.56 volumes of ethyl acetate, vortexing three times for $2 \mathrm{~min}$ and centrifuging for $5 \mathrm{~min}$ at 10,000 rpm (the pellet was discarded). The cell extraction was carried out by adding one volume of acetone, followed by sonication in an ultrasonic bath ( $2 \mathrm{~min}$ on, $2 \mathrm{~min}$ off; three cycles), and centrifugation at 10,000 rpm for $10 \mathrm{~min}$. The pellets were subjected to a second extraction using ethyl acetate (instead of acetone), following the same vortexing and centrifugation protocol, and the ethyl acetate supernatants were collected. The culture supernatant extracts (ethyl acetate) were vacuum-dried using rotary evaporation (RV 10 Digital, IKA®-Werke GmbH \& Co. KG, Germany). The cell extracts (acetone and ethyl acetate) were added to the same evaporating flasks in which the supernatant culture extracts were dried, and vacuum-dried by rotary evaporation. The dry extracts (from the supernatant cultures and the cells in the S. coelicolor, and only the supernatant cultures in the $S$. venezuelae) were kept at $-20{ }^{\circ} \mathrm{C}$ in the evaporating flasks.

The dry extracts were dissolved in $4 \mathrm{~mL}$ of methanol, transferred to 2-mL centrifugation tubes and dried in the speedvac. The pellets were dissolved and combined in $400 \mu \mathrm{L}$ of methanol:DMSO $(1: 1, v / v)$. Five $\mu \mathrm{L}$ from each sample were analysed by liquid chromatography-electrospray ionisation mass spectrometry (LC-HRESI-MS). A UPLC system (Dionex Ultimate 3000, Thermo Scientific, Waltham, MA, USA), coupled with an ESI-UHR-Qq-TOF Impact II spectrometer (Bruker, Billerica, MA, USA), was used, with negative ion mode acquisition in a $m / z$ range from 40 to $2000 \mathrm{Da}$. The chromatographic separation was carried out using a Zorbax ${ }^{\circledR}$ Eclipse Plus C18 column $(50 \times 2.1 \mathrm{~mm}, 1.8 \mu \mathrm{m})$ (Agilent Technologies, Santa Clara, CA, USA).

The $S$. coelicolor analytes were eluted at a flow rate of $0.25 \mathrm{~mL} / \mathrm{min}$ using a gradient of $0.1 \%(v / v)$ formic acid in water (mobile phase A) and $0.1 \%(v / v)$ formic acid in acetonitrile (mobile phase B), as follows: $0-10 \%(v / v)$ of B for $1 \mathrm{~min}$, which was increased to $35 \%$ over $3 \mathrm{~min}$, maintained at $35 \%$ for $1 \mathrm{~min}$, then increased to $100 \%$ over $3 \mathrm{~min}$, maintained at $100 \%$ during $2 \mathrm{~min}$, decreased to $10 \%$ over $1 \mathrm{~min}$ and maintained at $10 \%(v / v)$ for $4 \mathrm{~min}$.

The $S$. venezuelae analytes were eluted using the same flow rate and mobile phases as in the S. coelicolor, but the gradient was different: $0-5 \%(v / v)$ of B for $5 \mathrm{~min}$, which was increased to $55 \%(v / v)$ over $25 \mathrm{~min}$, and finally increased to $100 \%$ over $13 \mathrm{~min}$. 


\subsection{S. albus HPLC-DAD Metabolome Analyses}

The $S$. albus samples were collected from $108 \mathrm{~h}$ cultures. The compounds were extracted as indicated above for the S. coelicolor. The samples were injected into the Agilent 1260 Infinity instrument equipped with an analytical C- 18 HPLC column $(250 \times 4.0 \mathrm{~mm}$, Pursuit ${ }^{\circledR}$ XRs 5, $5 \mu \mathrm{m}$, Agilent Technologies, Santa Clara, CA, USA) and coupled to a UV detector set to $190-640 \mathrm{~nm}$ at a flow rate of $1 \mathrm{~mL} / \mathrm{min}$ over a period of $51 \mathrm{~min}$, using $\mathrm{H}_{2} \mathrm{O}$ (A) and $\mathrm{MeCN}(\mathrm{B})$ as the mobile phase, both with $0.1 \%(v / v)$ of formic acid: $0-5 \mathrm{~min}(10 \%$ B), $5-22 \min (10-35 \%$ B), $22-27 \min (35 \%$ B), $27-44 \min (35-100 \%$ B), $44-45 \min (100 \%$ B), $45-46 \min (100-10 \% \mathrm{~B})$ and $46-51 \mathrm{~min}(10 \% \mathrm{~B})$.

\subsection{Bioinformatic Analysis of the Metabolome}

The raw data were processed with the software package DataAnalysis (version 4.3, Bruker, Billerica, MA, USA ). The chromatograms were calibrated using internal calibrants (sodium, phosphate acetate). Both the culture media used for the fermentation contained MOPS, which creates an intense peak that is easily recognisable at the beginning of the chromatogram. The MOPS was used to test the reliability of the calibration, which had an average deviation of \pm 0.0002 (i.e., $0.2 \mathrm{ppm}$ ).

Chromatograms were processed using MZmine 2 software (G0 Cell Unit, Okinawa Institute of Science and Technology, Onna, Okinawa, Japan) [31]. Each sample was compared against the wild-type strain (three biological replicates each) according to the following workflow: mass detection was performed with the centroid mass detector using a noise level of 2.0E2; the ADAP chromatogram builder was set at a minimum group of 10, a group intensity threshold of 2.0E2, a minimum highest intensity of 5.0E2 and a $m / z$ tolerance of 0.001; smoothing was performed with a filter width of 9 ; the chromatogram deconvolution used a local minimum search, a chromatogram threshold of $90 \%$, a minimum RT range of 0.05 , a minimum relative height of $1 \%$, a minimum absolute height of $5.0 \mathrm{E} 2$, a minimum ratio of peak top/edge 2 and a peak duration range between 0.03 and $3, m / z$ center calculation median; the isotopes were grouped using a $m / z$ tolerance of 0.001 and an RT tolerance of 0.1 , a monoisotopic shape, a maximum charge of 2; the representative isotope was selected as the most intense; using a join aligner with a $m / z$ tolerance of 0.001 , weight for $m / z$ of 20, RT tolerance of 0.1 , weight for RT of 20, we compared the isotope pattern with a $m / z$ tolerance of 0.001 , minimum absolute intensity of 5.0E2 and a minimum score of $50 \%$; and the duplicate peak filter used the single feature filter mode, an $m / z$ tolerance of 0.001 and an RT tolerance of 0.1 .

The MZmine results were exported to MetaboAnalyst 5.0 (Institute of Parasitology, McGill University, Montreal, QC, Canada) [32] for statistical analysis. To focus on the most reliable data, abundance values lower than 1e4 were discarded before statistical analysis. The MetaboAnalyst parameters were as follows: the missing values were estimated as $1 / 5$ of the minimum positive value of each variable; the abundance values were $\log 10$ transformed; and the data were scaled by "pareto scaling". The differences in abundance values (compared to the wild-type strain) were considered significant if the q-value was equal to or less than 0.05 .

The secondary metabolites produced by the Streptomyces coelicolor M145 and the Streptomyces venezuelae NRRL B-65442 were deduced from Nett et al. [22] and Lee et al. [7] respectively. The formula and monoisotopic exact masses of these secondary metabolites were obtained from Pubchem (https:/ / pubchem.ncbi.nlm.nih.gov/) (accessed on 30 June 2021). The exact masses of the ions of these compounds and their putative adducts were obtained according to Huang et al. [33]. This list of secondary metabolite masses was used to identify the putative secondary metabolites in the S. coelicolor and S. venezuelae metabolomes, using a mass tolerance threshold of $\pm 0.001 \mathrm{Da}$. The retention time tolerance used to consider a specific mass as an isomer was $0.1 \mathrm{~min}$ (i.e., differences higher than 0.1 indicated different isomers). 


\section{Conclusions}

In this study, we show that increasing the cytosolic copper concentration by means of the alteration of the SCO2730/31 orthologue expression helps to modulate secondary metabolism in S. coelicolor, S. venezuelae and S. albus. The antisense SCO2730/31 consensus mRNA created here constitutes a tool for the knockdown of the expression of the SCO2730/31 genes which can contribute to the modification of secondary metabolism in streptomycetes and the activation of cryptic pathways, albeit to a lower level than in the S. coelicolor SCO2730::Tn5062 mutant. Further work will be necessary to fully understand the complex regulation of the SCO2730/31's gene expression and to reproduce the dose of the SCO2730/31 copper chaperone/transporter of the S. coelicolor SCO2730::Tn5062 mutant in other streptomycetes.

Supplementary Materials: The following are available online at https:/ /www.mdpi.com/article/10 .3390/ijms221810143/s1, Figure S1: SCO2730/31 antisense mRNAs; Table S1: Area of the compounds showing significant differences between the S. coelicolor harbouring pNG4-SP44, the S. coelicolor SCO2730/31 knockdown, the SCO2730::Tn5062 mutant, and the wild-type strain; Table S2: Area of the compounds showing significant differences between the S. venezuelae harbouring pNG4-SP44, the S. venezuelae SCO2730/31 knockdown, and the wild-type strain.

Author Contributions: N.G.-Q., I.G.-D.-R., P.G.-C., G.F.-G., S.A.-F., P.Y. and Á.P.-V. performed the experiments. P.G.-C. and M.M.-B. designed the copper quantification workflow. N.G.-Q. and Á.M. conceived the study. M.M.-B., F.L. and A.M. wrote the manuscript, with input from all of the authors. All authors have read and agreed to the published version of the manuscript.

Funding: This study was funded by the European Research Council (ERC PoC Strpcryptpath 835857), the "Ministerio de Ciencia, Innovación Universidades / Agencia Estatal de Investigación / Fondo Europeo de Desarrollo Regional" (RTI2018-093978-B-I00) and the "Consejería de Empleo, Industria y Turismo del Principado de Asturias" (FC-GRUPIN-IDI/2018/000120). Gemma Fernández-García was funded by a pre-doctoral grant from the "Universidad de Oviedo". Sergio Alonso and Ignacio Gutiérrez-Del-Río were funded by two "Severo Ochoa" predoctoral grants from "Consejería de Ciencia, Innovación y Universidad del Principado de Asturias".

Data Availability Statement: The LC-MS raw data are available upon request.

Acknowledgments: We are grateful to the technical support provided by "Servicios CientíficoTécnicos de la Universidad de Oviedo".

Conflicts of Interest: The authors declare no conflict of interest.

\section{References}

1. Berdy, J. Bioactive microbial metabolites. J. Antibiot. 2005, 58, 1-26. [CrossRef]

2. Hopwood, D.A. Streptomyces in Nature and Medicine: The Antibiotic Makers; Oxford University Press: New York, NY, USA; Oxford, UK, 2007.

3. Flardh, K.; Buttner, M.J. Streptomyces morphogenetics: Dissecting differentiation in a filamentous bacterium. Nat. Rev. Microbiol. 2009, 7, 36-49. [CrossRef]

4. Yague, P.; Lopez-Garcia, M.T.; Rioseras, B.; Sanchez, J.; Manteca, A. Pre-sporulation stages of Streptomyces differentiation: State-of-the-art and future perspectives. FEMS Microbiol. Lett. 2013, 342, 79-88. [CrossRef]

5. Xia, H.; Zhan, X.; Mao, X.M.; Li, Y.Q. The regulatory cascades of antibiotic production in Streptomyces. World J. Microbiol. Biotechnol. 2020, 36, 13. [CrossRef] [PubMed]

6. Onaka, H. Novel antibiotic screening methods to awaken silent or cryptic secondary metabolic pathways in actinomycetes. J. Antibiot. 2017, 70, 865-870. [CrossRef]

7. Lee, N.; Kim, W.; Hwang, S.; Lee, Y.; Cho, S.; Palsson, B.; Cho, B.K. Thirty complete Streptomyces genome sequences for mining novel secondary metabolite biosynthetic gene clusters. Sci. Data 2020, 7, 55. [CrossRef] [PubMed]

8. Manteca, A.; Yague, P. Streptomyces Differentiation in Liquid Cultures as a Trigger of Secondary Metabolism. Antibiotics 2018, 7, 41. [CrossRef] [PubMed]

9. Zhang, Z.; Du, C.; de Barsy, F.; Liem, M.; Liakopoulos, A.; van Wezel, G.P.; Choi, Y.H.; Claessen, D.; Rozen, D.E. Antibiotic production in Streptomyces is organized by a division of labor through terminal genomic differentiation. Sci. Adv. 2020, 6, eaay5781. [CrossRef]

10. Nguyen, C.T.; Dhakal, D.; Pham, V.T.T.; Nguyen, H.T.; Sohng, J.K. Recent Advances in Strategies for Activation and Discovery/Characterization of Cryptic Biosynthetic Gene Clusters in Streptomyces. Microorganisms 2020, 8, 616. [CrossRef] 
11. Keijser, B.J.; van Wezel, G.P.; Canters, G.W.; Kieser, T.; Vijgenboom, E. The ram-dependence of Streptomyces lividans differentiation is bypassed by copper. J. Mol. Microbiol. Biotechnol. 2000, 2, 565-574.

12. Ueda, K.; Tomaru, Y.; Endoh, K.; Beppu, T. Stimulatory effect of copper on antibiotic production and morphological differentiation in Streptomyces tanashiensis. J. Antibiot. 1997, 50, 693-695. [CrossRef] [PubMed]

13. Gonzalez-Quinonez, N.; Corte-Rodriguez, M.; Alvarez-Fernandez-Garcia, R.; Rioseras, B.; Lopez-Garcia, M.T.; Fernandez-Garcia, G.; Montes-Bayon, M.; Manteca, A.; Yague, P. Cytosolic copper is a major modulator of germination, development and secondary metabolism in Streptomyces coelicolor. Sci. Rep. 2019, 9, 4214. [CrossRef]

14. Fujimoto, M.; Yamada, A.; Kurosawa, J.; Kawata, A.; Beppu, T.; Takano, H.; Ueda, K. Pleiotropic role of the Sco1/SenC family copper chaperone in the physiology of Streptomyces. Microb. Biotechnol. 2012, 5, 477-488. [CrossRef]

15. Liu, X.; Tang, J.; Wang, L.; Liu, R. Mechanism of CuO nano-particles on stimulating production of actinorhodin in Streptomyces coelicolor by transcriptional analysis. Sci. Rep. 2019, 9, 11253. [CrossRef] [PubMed]

16. Worrall, J.A.; Vijgenboom, E. Copper mining in Streptomyces: Enzymes, natural products and development. Nat. Prod. Rep. 2010, 27, 742-756. [CrossRef] [PubMed]

17. Straw, M.L.; Chaplin, A.K.; Hough, M.A.; Paps, J.; Bavro, V.N.; Wilson, M.T.; Vijgenboom, E.; Worrall, J.A.R. A cytosolic copper storage protein provides a second level of copper tolerance in Streptomyces lividans. Metallomics 2018, 10, 180-193. [CrossRef]

18. Kieser, T. Practical Streptomyces Genetics; John Innes Foundation: Norwich, UK, 2000.

19. Ehrlich, J.; Bartz, Q.R.; Smith, R.M.; Joslyn, D.A.; Burkholder, P.R. Chloromycetin, a New Antibiotic From a Soil Actinomycete. Science 1947, 106, 417. [CrossRef] [PubMed]

20. Myronovskyi, M.; Rosenkranzer, B.; Nadmid, S.; Pujic, P.; Normand, P.; Luzhetskyy, A. Generation of a cluster-free Streptomyces albus chassis strains for improved heterologous expression of secondary metabolite clusters. Metab. Eng. 2018, 49, 316-324. [CrossRef]

21. Bai, C.; Zhang, Y.; Zhao, X.; Hu, Y.; Xiang, S.; Miao, J.; Lou, C.; Zhang, L. Exploiting a precise design of universal synthetic modular regulatory elements to unlock the microbial natural products in Streptomyces. Proc. Natl. Acad. Sci. USA 2015, 112, 12181-12186. [CrossRef]

22. Nett, M.; Ikeda, H.; Moore, B.S. Genomic basis for natural product biosynthetic diversity in the actinomycetes. Nat. Prod. Rep. 2009, 26, 1362-1384. [CrossRef]

23. Stuttard, C. Temperate Phages of Streptomyces venezuelae: Lysogeny and Host Specificity Shown by Phages SV1 and SV2. Microbiology 1982, 128, 115-121. [CrossRef]

24. Novella, I.S.; Barbes, C.; Sanchez, J. Sporulation of Streptomyces antibioticus ETHZ 7451 in submerged culture. Can. J. Microbiol. 1992, 38, 769-773. [CrossRef] [PubMed]

25. Sanchez, C.; Butovich, I.A.; Brana, A.F.; Rohr, J.; Mendez, C.; Salas, J.A. The biosynthetic gene cluster for the antitumor rebeccamycin: Characterization and generation of indolocarbazole derivatives. Chem. Biol. 2002, 9, 519-531. [CrossRef]

26. Rioseras, B.; Yague, P.; Lopez-Garcia, M.T.; Gonzalez-Quinonez, N.; Binda, E.; Marinelli, F.; Manteca, A. Characterization of SCO4439, a D-alanyl-D-alanine carboxypeptidase involved in spore cell wall maturation, resistance, and germination in Streptomyces coelicolor. Sci. Rep. 2016, 6, 21659. [CrossRef] [PubMed]

27. Tsao, S.W.; Rudd, B.A.; He, X.G.; Chang, C.J.; Floss, H.G. Identification of a red pigment from Streptomyces coelicolor A3(2) as a mixture of prodigiosin derivatives. J. Antibiot. 1985, 38, 128-131. [CrossRef] [PubMed]

28. Bystrykh, L.V.; Fernandez-Moreno, M.A.; Herrema, J.K.; Malpartida, F.; Hopwood, D.A.; Dijkhuizen, L. Production of actinorhodin-related "blue pigments" by Streptomyces coelicolor A3(2). J. Bacteriol. 1996, 178, 2238-2244. [CrossRef] [PubMed]

29. Bradford, M.M. A rapid and sensitive method for the quantitation of microgram quantities of protein utilizing the principle of protein-dye binding. Anal. Biochem. 1976, 72, 248-254. [CrossRef]

30. Manteca, A.; Alvarez, R.; Salazar, N.; Yague, P.; Sanchez, J. Mycelium differentiation and antibiotic production in submerged cultures of Streptomyces coelicolor. Appl. Environ. Microbiol. 2008, 74, 3877-3886. [CrossRef] [PubMed]

31. Pluskal, T.; Castillo, S.; Villar-Briones, A.; Oresic, M. MZmine 2: Modular framework for processing, visualizing, and analyzing mass spectrometry-based molecular profile data. BMC Bioinform. 2010, 11, 395. [CrossRef]

32. Pang, Z.; Chong, J.; Zhou, G.; de Lima Morais, D.A.; Chang, L.; Barrette, M.; Gauthier, C.; Jacques, P.E.; Li, S.; Xia, J. MetaboAnalyst 5.0: Narrowing the gap between raw spectra and functional insights. Nucleic Acids Res. 2021. [CrossRef]

33. Huang, N.; Siegel, M.M.; Kruppa, G.H.; Laukien, F.H. Automation of a Fourier transform ion cyclotron resonance mass spectrometer for acquisition, analysis, and e-mailing of high-resolution exact-mass electrospray ionization mass spectral data. $J$. Am. Soc. Mass Spectrom. 1999, 10, 1166-1173. [CrossRef] 